\title{
Chromomeres revisited
}

\author{
Herbert C. Macgregor
}

Published online: 7 September 2012

(C) Springer Science+Business Media B.V. 2012

\begin{abstract}
The history of studies on the chromomeres of lampbrush chromosomes is outlined and evidence for the nature and function of these structures is collected and summarised. Chromomeres and their associated loops on lampbrush chromosomes are not genetic units although in some special cases, they consist of specific families of repeated DNA sequences. The emergence of a chromomeric organisation coincides with the onset and intensification of transcription on lampbrush loops. Modern molecular studies have provided evidence that the chromatin of lampbrush chromomeres differs in several important respects from that of condensed metaphase chromosomes. It is in a highly dynamic state that facilitates localised transcription whilst keeping the chromosome safe from structural changes that might impede its orderly progression up to and through meiotic metaphase 1. Lampbrush chromosomes (LBCs) are a physically induced phenomenon, facilitated by the selective absence of molecular factors that would interfere with their main transcriptional role. LBC morphology is highly dynamic and driven by transcriptive activity.
\end{abstract}

Responsible Editor: Conly Rieder

H. C. Macgregor $(\bowtie)$

School of Biosciences, University of Exeter,

Exeter EX4 4SB, UK

e-mail: herbert.macgregor@btinternet.com
Keywords lampbrush chromosome - chromomeres chromatin $\cdot$ transcription
Abbreviations
bp
Base pairs
CENP-A Centromeric protein A
DAPI 4',6-Diamidino-2-phenylindole
LBC Lampbrush chromosome
H4 Histone H4
H3K4me3 A modified histone H3 that marks transcriptional activation
HMGN High molility group nucleosome binding proteins
HP A protein involved in heterochromatin formation and gene silencing
SMC Structural maintenance of chromosome protein
RAD21 A protein involved in chromosome condensation in mitosis
XCAP-D2 A condensin complex with a role in chromosome condensation and segregation

\section{In the beginning}

In the autumn of 1958, Mick Callan (Fig. 1) asked me to make some preparations of the lampbrush chromosomes from a female crested newt, fix them and stain them with leuco-basic fuchsin after a mild treatment with hydrochloric acid - the "Feulgen Reaction". If the tiny granules - chromomeres - that were distributed along the 


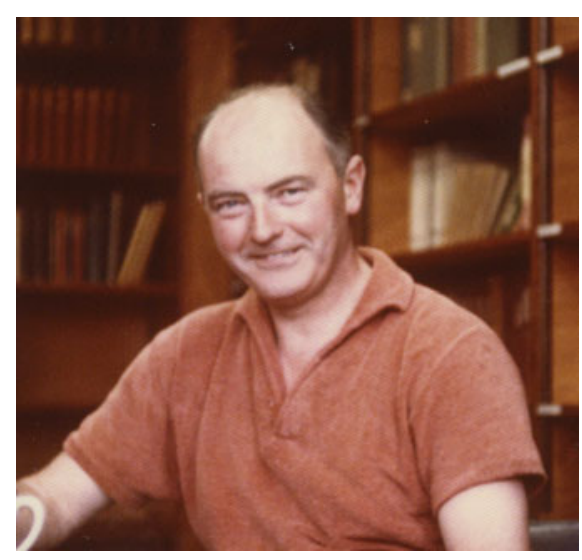

Fig. 1 Professor HG (Mick) Callan FRS photographed in 1965 in his laboratory in the University of St. Andrews, Scotland

axes of these chromosomes contained DNA, they should stain pink. It was a tough assignment for a fresh post-graduate, and it took me 2 weeks accomplish. The chromomeres were tiny: just visible with a carefully adjusted microscope fitted with a $100 \times$ flat field oil immersion objective. With a bit of imagination and self-persuasion, they were pink - just - their contrast made more convincing with a green filter. But yes, they were definitely pink and with some critical controls built in, there could be no reasonable doubt that they contained DNA. For more than half a century, these tiny granules and all the many dogmas and ideas surrounding them have bothered me. What are they and how do they fit into the context of life?

\section{Historical}

In the third edition of his monumental book, Wilson (1925) cites studies on spermatocyte nuclei of 10 animals from five different phyla and defines chromomeres as "....spireme threads which in some sense or other are serial aggregates which have a perfectly definite organisation and one that differs specifically from chromosome to chromosome and from species to species. Chromomeres are capable not only of growth, definite alignment and division, but also of conjugating two by two and like with like".

Twenty-nine years later, Michael White (1954) says of chromomeres that they are "....distinctly staining with a definite and species-specific linear pattern". He cites observations on meiotic prophase chromosomes and lampbrush chromosomes and concludes that they are "....... a universal constituent of the chromosomes and each ultimate chromomere corresponds to a single genetic locus". Bear in mind that in 1954, lampbrush chromosome technology was in its infancy and White was probably overstepping the evidence that was available at that time.

Then after another 21 years, Vlad and Macgregor (1975), on the basis of experimental evidence and in the context of a wide-ranging review of everything that had been observed about chromomeres up to 1975, concluded that "lampbrush chromomeres are not the genetic units they were once supposed to be and their formation must be related to their DNA sequence content".

Callan (1986; Fig. 1), focussing specifically on lampbrush bivalents, reported that "......chromomeric patterns are rarely identical in the two homologues", which was surprising and somewhat perplexing at the time, in view of the strikingly regular species specificity of chromomeric (=band) pattern in polytene chromosomes from dipteran flies.

So at the end of the twentieth century, we have, on the one hand, the long established and well-known situation in polytene chromosomes where chromomeres form a regular linear pattern that bears some relationship to the arrangement of genes along the length of a chromosome. On the other hand, we have LBCs where there are apparent discrepancies with regard genetic equivalence, with just a hint of a constant pattern that may be complicated on lampbrush chromosomes by widespread transcription and by stretching as a result of the punishment they receive when being isolated by free hand dissection from the germinal vesicle. And then we have spermatocytes, the original source from which chromomeric organisation was first described by Wilson nearly 100 years ago.

The basis of polytene chromomeres is a matter for another day, except to say that the fact that they line up transversely to form bands is unremarkable, since each chromatid is an undisplaced identical endomitotic replica and there are many of them.

Why are spermatocyte pachytene chromosomes "chromomeric"? Or are they? Or maybe they are more like lampbrushes than we think and have some transcriptive business to attend to - a matter on which the sparse existing evidence remains decidedly equivocal.

What I am concentrating on here are the lampbrush chromosomes of animal oocytes which must surely 
rank amongst the most amazingingly beautiful and intricate objects of the entire microscopic world.

\section{Some facts about lampbrush chromosomes and their chromomeres}

The very first thing to say about lampbrush chromosomes-and it is something that people who have never seen them have difficulty in grasping - is that the nucleus in which they reside is enormous (Fig. 2) and the chromosomes themselves are really huge (Fig. 3). Urodele amphibians, which have provided much of the material for past LBC research, have some of the largest genomes of all organisms. The biggest urodele chromosome, found in a tiny Central American salamander, is nearly 2,000 times the size of the fourth chromosome of Drosophila melanogaster. The situation was discussed by me (Macgregor 1978) and redefined by Sessions (2008).

In the female's gametocytes, these big chromosomes go from a compact telophase form at the end of the last oogonial mitosis, become lampbrushy and then contract again to form normal-looking first

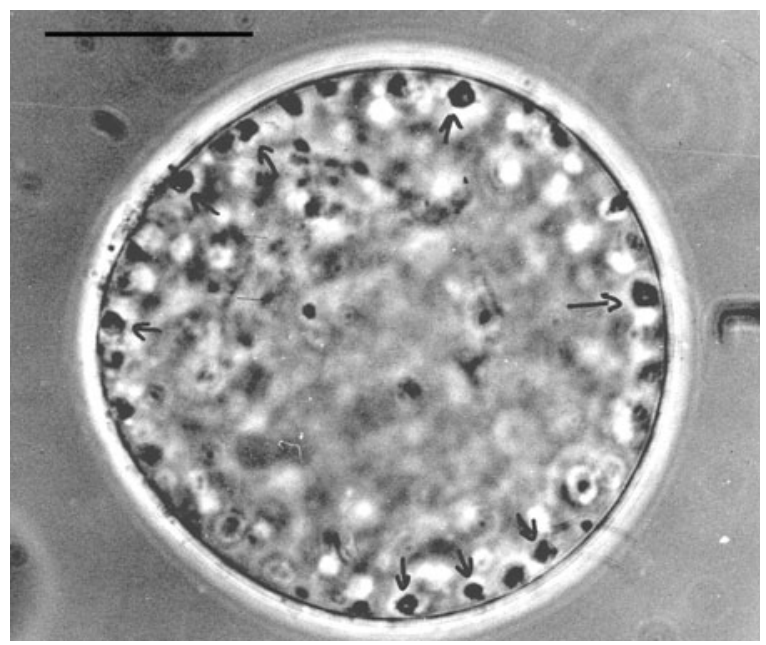

Fig. 2 A nucleus freshly isolated by hand from a newt oocytea germinal vesicle - and seen here by phase contrast, unfixed and life-like, in a physiologically balanced saline solution. The oocyte from which this nucleus came was $500 \mu \mathrm{m}$ in diameter. The scale on this micrograph corresponds to $50 \mu \mathrm{m}$. The arrows indicate some of the many hundreds of nucleioli that represent the consequences of the massive amplification of ribosomal genes and that contribute to the busy and crowded environment in this germinal vesicle. Most of the inner part of the nucleus is occupied by the 12 lampbrush bivalents

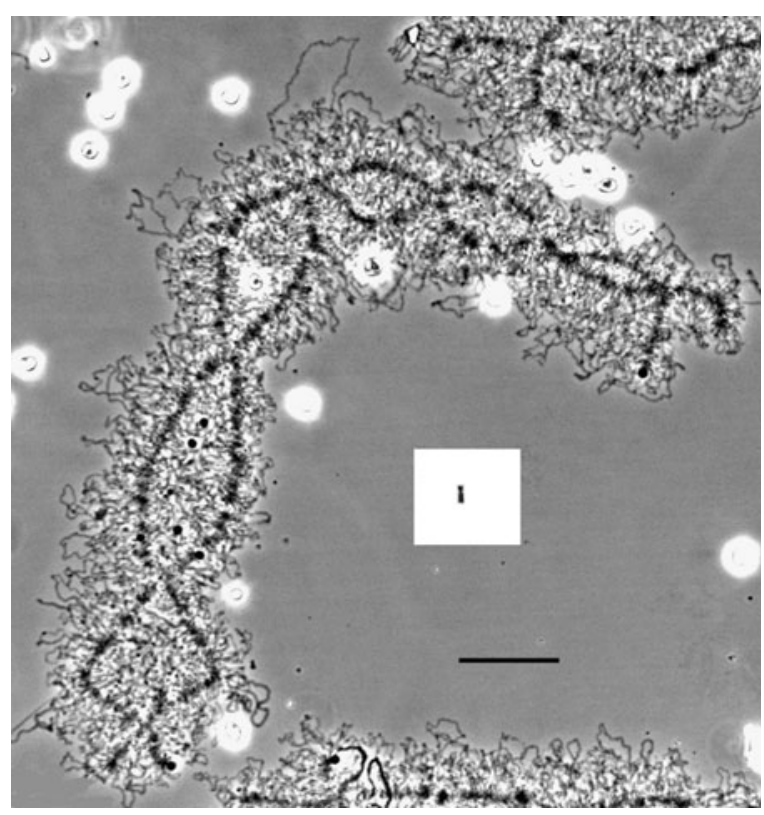

Fig. 3 This photograph shows a medium-sized lampbrush bivalent freshly isolated from an axolotl (Ambystoma mexicanum) oocyte and viewed in its life-like form with phase contrast microscopy. As a comparison, the inset shows at the same magnification a medium-sized mitotic metaphase chromosome from an axolotl larva. That the relatively minute volume of chromatin represented by the metaphase chromosome should be so transformed and rearranged as to constitute the DNA axis of a lampbrush chromosome that is nearly a millimetre long and all its lateral loops signifies that something very special must be happening on these amazing chromosomes. (Photograph courtesy of the late HG Callan FRS and Dr. Garry Morgan of Nottingham University UK.) Scale bar $50 \mu \mathrm{m}$

meiotic metaphase bivalents. The vast difference in size between a chromosome when it is a full blown lampbrush (actually visible to the naked eye!) and the same chromosome when it is in metaphase (Fig. 3) must always be kept in mind when considering any aspect of lampbrush structure and function. The change from one form to the other represents one of nature's most dramatic supramolecular metamorphoses.

Lampbrush chromosomes are meiotic bivalents, so each half-bivalent (=homologue) consists of two chromatids, making up a structure that is diagrammatically represented in Fig. 4. The reader can learn all about them, the work that has been done on them and the people who did that work from the website http:// www.projects.exeter.ac.uk/lampbrush .

The currently accepted model of lampbrush organisation (Fig. 4), emphasises the two chromatid structure of the chromosome, a chromomere that must 


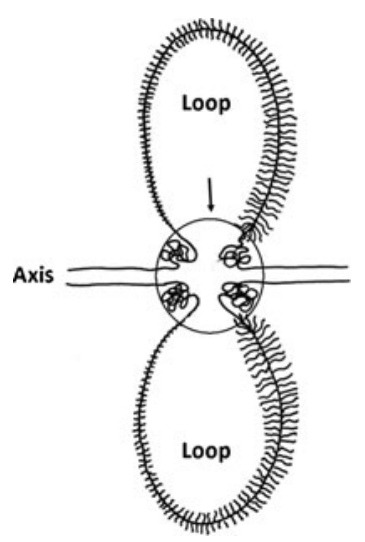

Fig. 4 A diagram of the basic organisation of a lampbrush chromosome, showing the two chromatids that form the main axis of the chromosome and their lateral separation to form two loops. The area contained within the circle (arrow) is the chromomere, represented here hypothetically as comprising four regions of non-transcribed chromatin, a scheme that is not inconsistent with a range of observations that are outlined later

comprise four parts held together in some way without intimate intertwining of the chromatin fibres in each part and sister loops that have similar, though not necessarily identical lengths and the same directions of thickness polarity.

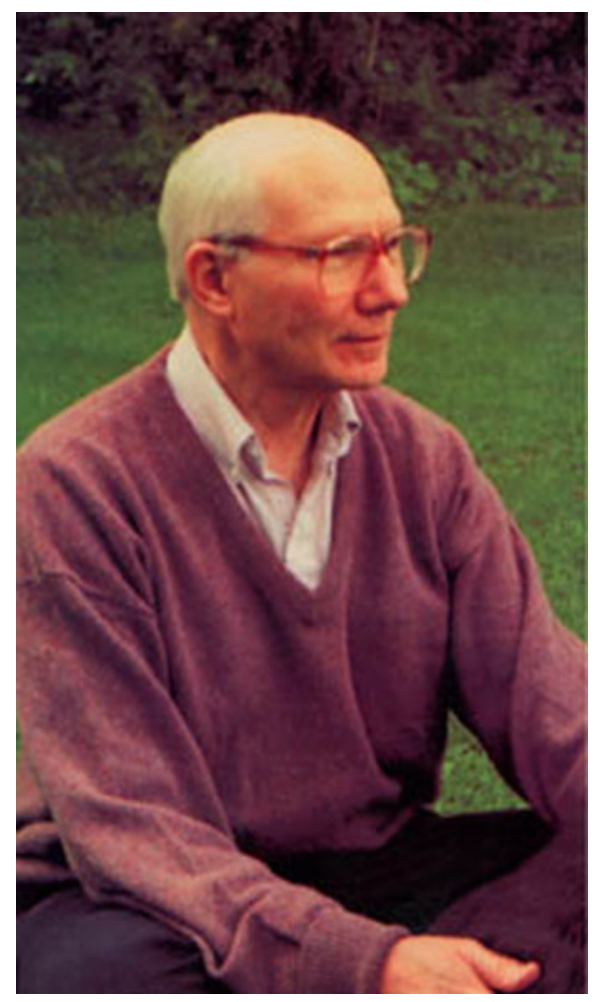

Fig. 5 Joseph G Gall (Joe) photographed in 1998
It deserves special mention here, even if it has little to do with chromomeres, that the basis of this modelan interchromomeric fibre consisting of two DNA double helices with loops consisting of just one double helix - was convincingly proved by an experiment carried out by Joe Gall (Fig. 5) in 1963 that involved just a simple light microscope, a stopwatch and a little bit of elementary mathematics. If you have not already done so, I recommend that you find and read this classical gem of chromosome science (Gall 1963).

\section{Lampbrush chromomeres are real}

They occur all along all of the chromosomes and they range in size from barely resolvable to about $1 \mu \mathrm{m}$ (Fig. 6). An animal with a haploid genome size $7 \times$ that of the human genome (=ca. 21 billion base pairs (bp)) has a total of about 5,000 chromomeres in a haploid set of its fully developed lampbrush chromosomes. Chromomeres stain with the Feulgen reaction and with 4',6-diamidino-2-phenylindole (DAPI; Fig. 7) and look like blobs of chromatin in electron micrographs of sections of fixed and embedded LBCs (Mott and Callan 1975). No surprises! An average lampbrush chromomere from a urodele amphibian contains between 5 and $10 \mathrm{Mb}$ of DNA, bearing in mind that it is derived from two chromatids. Loops have been reckoned to have between 50 and $150 \mathrm{~kb}$.

According to the classical lampbrush dogma, laid down in the 1960s by two of the modern lampbrush pioneers, Joe Gall and Mick Callan, most chromomeres

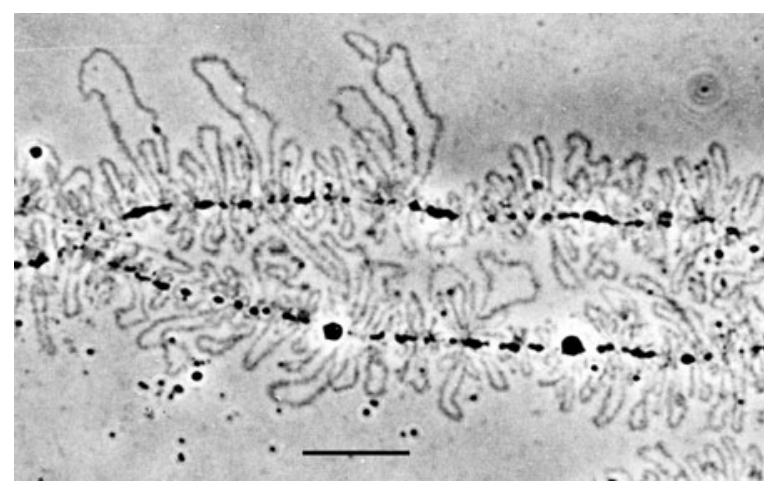

Fig. 6 Phase contrast micrograph of part of a lampbrush bivalent that has been isolated in a physiological saline and then centrifuged lightly to flatten all the loops down on the surface of the isolation chamber, so making the chromosome axis and its chromomeres more easily seen. The scale represents $20 \mu \mathrm{m}$ 

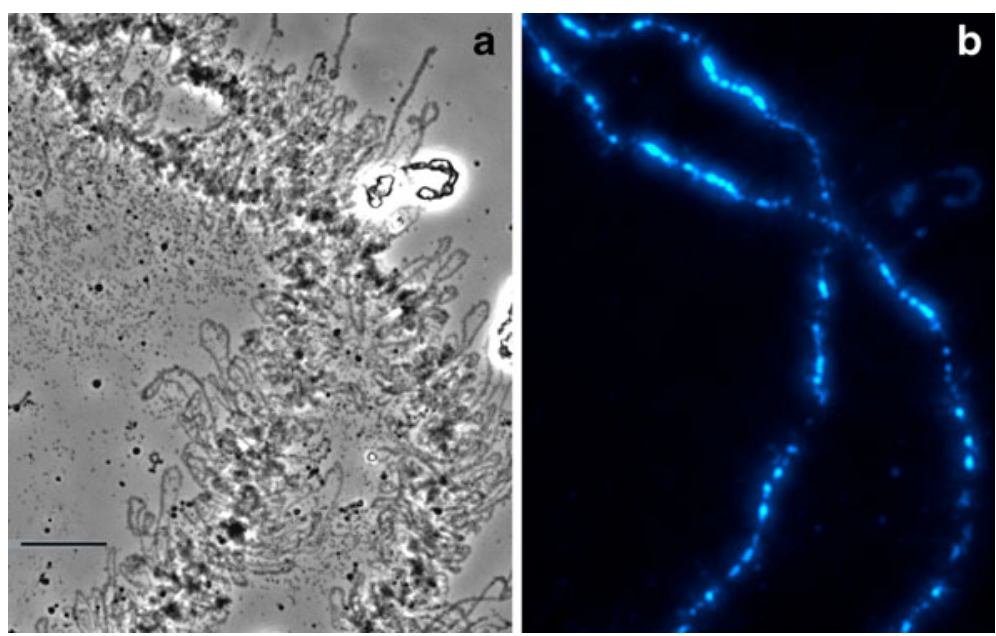

Fig. 7 a Part of a freshly isolated lampbrush bivalent from the axolotl (Ambystoma mexicanum) photographed in phase contrast. b The same part of the same bivalent after fixation, staining with DAPI and photographed with a fluorescence microscope. Three matters are noteworthy: 1 the axes of the half-bivalents are clearly defined by their DAPI staining, 2

are resolvable at the light microscope level as discrete objects joined to one another by a very thin interchromomeric fibre and each chromomere has one or several pairs of lampbrush loops springing from it. All absolutely true.

The sizes of chromomeres were said to be related to the lengths of the loops associated with them, which was the basis of the (later discredited) spinning out and retraction hypothesis for lampbrush function (Snow and Callan 1969). The main evidence for this relationship is derived from the fact that at the end of the lampbrush phase of meiosis, the chromosomes contract, the chromomeres become fewer and larger and the loops become shorter. But let us be clear about one thing, no objective study has ever been carried out to test this relationship. It may not be true and our thoughts about LBC make-up are no longer constrained by it.

\section{Is there a chromomeric pattern?}

In considering whether the chromomeres of LBCs do conform to a locus-specific pattern, I will cite just three lines of evidence.

First, the W chromosome of a chicken (Gallus domesticus) consists of a small number of large chromomeres that form a distinct pattern (Solovei et al. 1993, 1998). Secondly, the very beautiful and
DAPI-stained chromomeres vary widely in size and 3 there is some approximate correspondence between the patterns and distribution of chromomeres on the two half-bivalents. The scale bar represents $20 \mu \mathrm{m}$. These micrographs were kindly provided by Garry Morgan, University of Nottingham, UK

exceedingly accurate drawings of LBCs that illustrate the Callan and Lloyd Royal Society paper (Callan and Lloyd 1960) about the LBCs of crested newts show, in just a few places, an almost precise correspondence between the chromomere pattern on homologous halfbivalents but in general, the chromomeric pattern of homologous half-bivalents does not match. Thirdly, in LBCs that are past the stage of maximum lampbrush development, the arrangement of chromomeres is definitely not identical on homologous half-bivalents.

From these observations, I conclude that if we were able to resolve and map the arrangement of chromomeres on LBCs in their truly life-like condition and at the stage of the maximum development of their loops -and hence the transcriptive activity in the germinal vesicle - a regular, chromosome- and species-specific pattern might be evident. But this pattern is usually obscure and whether or not it ever exists is not crucial to an understanding of LBCs.

\section{Chromomeres and genes}

What about chromomeres and genes? The immediate response to this must always be Mick Callan's clever observation on certain loci on the LBCs of crested newts where there are very large and conspicuous loops. In 1956, which is the veritable early dawn of 
modern lampbrushology, Callan and Lloyd (1956) noticed that these loops could be present or absent in different homozygous or heterozygous combinations. The pattern of presence or absence was the same in every oocyte from any one individual but varied from newt to newt. Later on, and in time for publication in his historic paper (Callan and Lloyd 1960), he showed that if presence or absence for these marker loops were treated as Mendelian characters, they assorted and combined within a population according to HardyWeinberg rules. So some "landmark loops" do bear some relationship to genes in the classical sense. Remember that here we are talking not about chromomeres but about distinctive loops (=transcription complexes) that arise from chromomeres.

So is the loop and its chromomere to be regarded as a genetic unit? Probably not. In the first place, chromomeres that have several pairs of loops associated with them are not uncommon. In bird LBCs, it has been observed that some chromomeres have lots of micro-loops associated with them (Gaginskaya and Tsvetkov 1988, Alla Krasikova unpublished observations). There are, moreover, many examples of loops that consist of several transcription units arranged in tandem springing from a single chromomere (Scheer et al. 1976) These observations would seem to prove that the DNA of chromomeres can be functionally, if not genetically heterogeneous.

Then there are the observations on chromomere number. Two quite closely related species in the same genus, identical karyotypes in terms of relative sizes and shapes of the chromosomes, species A has a genome twice as large as species B. The LBCs of A are twice as long as those of $\mathrm{B}$, yet at corresponding stages of oocyte development and LBC activity, the distribution density of chromomeres is the same on the LBCs of both. So, unquestionably, the LBCs of A have many more chromomeres than those of B (Vlad and Macgregor 1975).

No, the chromomeres of lampbrush chromosomes are not genetic units.

But are they structural units whose existence owes something to the physical and molecular make-up of the chromosomes, perhaps evolved in connection with the advantage of keeping two chromatids tightly associated and in strict register with one another during the intricate processes of meiotic synapsis, crossing over and segregation? Very possibly, in principle, but again the word "unit" does cause some problems.

\section{The physical properties of chromomeres}

When do chromomeres first appear? We do not know. Whilst there is plenty of information about transcription, there is almost none about chromomeres. LBCs from newt oocytes between 400 and 1,000 $\mu \mathrm{m}$ diameter have a chromomeric axis (Mott and Callan 1975). Ooctyes in this size have fully formed LBCs. There are well-developed LBCs in Xenopus oocytes of 300$450 \mu \mathrm{m}$ diameter (Hill and Macgregor 1980). Lampbrush-type transcription, however, starts much earlier in oocytes of less than $100 \mu \mathrm{m}$ diameter in Xenopus, from which it is exceedingly difficult to isolate by hand anything that resembles a lampbrush chromosome or see any evidence of such a thing. Are transcription and the emergence of a chromomeric organisation coincidental?

We know what the chromosomes look like in pachytene oocytes from Xenopus and we know what they look like in early diplotene oocytes from Triturus as they emerge from the pachytene stage and the germinal vesicle starts to enlarge (see Macgregor 1972). In both cases, they are short, condensed and loopless. What happens in the transformation from that to the fully developed lampbrush form is a steady build up in the number of transcription units and the degree to which they are loaded with RNA polymerase. So probably yes: the start of transcription and the breakup of condensed axis into a chromomeric arrangement go hand in hand; and both happen quite quickly. But the chromosome is never at any stage allowed to become fully decondensed.

Most of the older generation of lampbrushers have at one time or another looked at sections of chromomeres with a transmission electron microscope, but only Mott and Callan (1975) ever published what they found-and it is rather interesting. They say that "Most of the chromomeres in thin section show a characteristic basket-work texture, the densely staining material, presumably skeins of DNP fibrils, being some $30 \mathrm{~nm}$ wide and separated by spaces of some $50 \mathrm{~nm}$ wide ..... As well as the material of basket work texture, some chromomeres contain regions where the stained material is much more densely packed ..... In $2 \mathrm{~mm}$ oocytes (which are nearing the end of the LBC phase), to our amazement, we failed to find any trace of objects having a basket work texture. However, we were able to find objects having the densely packed fibrillar texture, reminiscent of the exceptional dense 
portions of chromomeres in spread preparations (of earlier oocytes)". In my view, the significance of these observations is that the chromomeres of transcriptionally active LBCs have a different, looser, texture than that of condensed chromatin, whilst in a few chromomeres there may be small localised regions that have remained fully condensed or signify the start of full condensation in the run-up to first meiotic metaphase. In a moment of perhaps misplaced modesty, Mott and Callan remark that the "basket-work" texture seen in their preparations is "evidently a preparative artefact". Yet their methods were careful, critical and were, at the time, likely to achieve the best possible structural preservation of chromosomal DNP. There is lots of evidence to suggest that what they saw was a true indication that there are indeed two physical states of chromatin whose existence is related to localised transcriptive activity.

Another, highly subjective matter may be relevant here. Those who have seen a DAPI-stained LBC and its metaphase counterpart will have is absolutely no shadow of doubt that the total DAPI fluorescence of the $\mathrm{LBC}$, confined to the bright little chromomeres, adds up to much more than that of the metaphase chromosome. Somebody should measure it sometime!

Now before anyone accuses me of sacrilege or senility, let me proclaim loudly that I do not intend to imply that a lampbrush chromatid has more DNA than its metaphase counterpart, but I would suggest that these subjective assertions may offer a clue to a special arrangement of LBC chromatin which results in its looser fine structure, an expanded chromomere volume and perhaps a greater affinity for or accessibility to DAPI.

One of the earliest and most significant observations on LBCs was the consequence of stretching the chromosomes during the very delicate free-hand process of isolating them from the oocyte nucleus. It is, in fact, extremely difficult to make a preparation of a set of LBCs without stretching or damaging them in one way or another; but it can be done. Upon being stretched longitudinally (and Callan did this deliberately and very skilfully in 1955 with a micromanipulator), the weakest point proves to be a transverse plane across the chromomere such that the loops attached to the chromomere come to span the gap between the two clearly discernible half-chromomeres (Figs. 8 and 2 of 9). Curiously enough, Callan's very first description of this was when he was looking at the

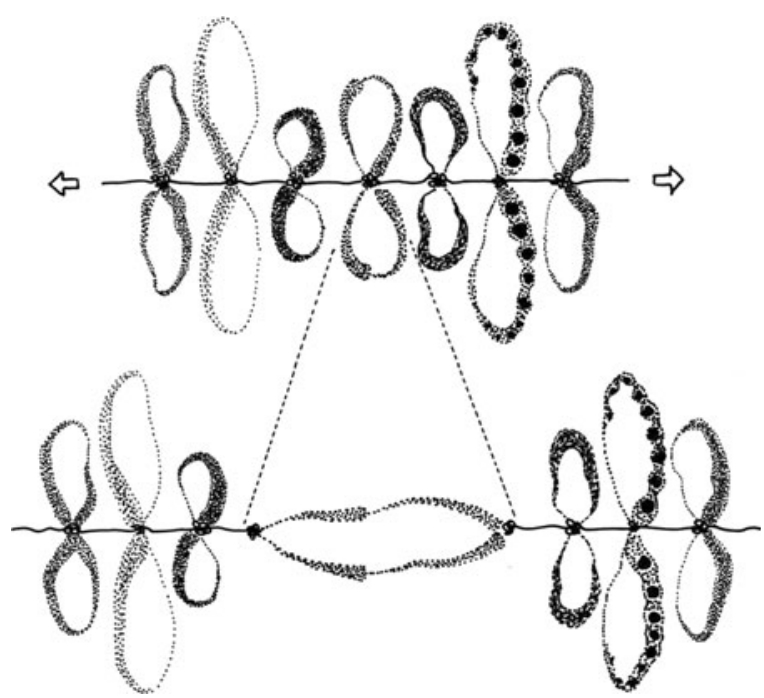

Fig. 8 The upper of the two drawings shows a portion of a lampbrush chromosome that is unstretched. The lower drawing shows the same region after being stretched longitudinally. The chromomere of the middle of the seven loop pairs shown has split transversely and its two loops bridge the gap between the two halves of the chromomere. The loops forming the double bridge consist of two tandemly arranged transcription units with similar, left to right, polarity of transcription

LBCs of the European marbled newt and also of an isopod, a mollusc and an elasmobranch (Callan 1957). So there would seem to be a plane of weakness across a chromomere in which two bundles of compacted chromatin are not intertwined or inseparable. To which I will add the aside that it suggests that transcription

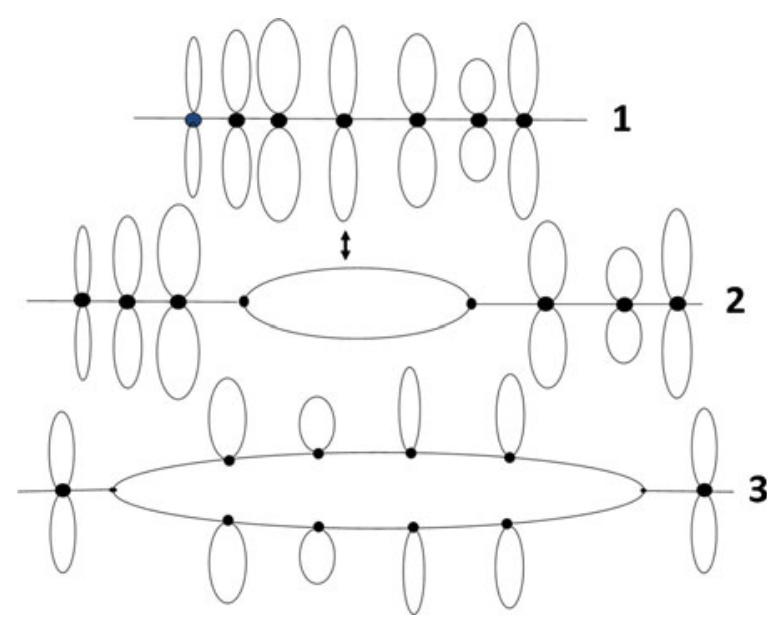

Fig. 91 Normal lampbrush organisation, chromomeres with paired loops. 2 Double loop bridge formed by stretching the chromosome. 3 Double-axis region with chromomeres bearing single loops 
starts before the chromomere is formed, since the loop defines the position of the chromomere.

What is more, it seems likely that there is a longitudinal plane of weakness as well as a transverse one. In specific locations along the LBCs of most of the vertebrates in which they have been studied, the chromosome axis is double. Each chromatid that makes up a half-bivalent has chromomeres that have just one loop associated with them (3 of Fig. 9). The more conspicuous of these "double-axis regions" are always in the same place and some of them feature in the LBC maps in the paper of Callan and Lloyd (1960) on the LBCs of crested newts. They represent places where the half-chromomeres are not fused with one another, even though their counterparts are just a short distance away. A conspicuous feature of Lydia Lloyd's meticulous drawings of double-axis regions (Callan and Lloyd 1960) is that the pattern of chromomeres and loops on each half of a double-axis region do correspond exactly. Does this perhaps signify a precise synchrony of the transcriptional events on the two identical chromatids, a synchrony which is not evident for homologous regions of two half-bivalents?

The most amazing example of "half LBCs" that have chromomeres with just one loop are the LBCs that form when sperm nuclei from frogs or from humans are injected into amphibian germinal vesicles, visualised in a series of landmark experiments from the Gall laboratory in 1998 (Gall and Murphy 1998, see also http:// projects.exeter.ac.uk/lampbrush/pics.htm). Sperm chromosomes, of course, consist of just one chromatid.

All these simple light microscope observations could perhaps be explained on the basis of there being certain specific factors that differentiate the chromatin of these discrete objects that we call chromomeres. Such factors would have to be fine tuned to differentiate between neighbouring chromomeres, hence the beads on a string appearance of an LBC and between the chromatin of the two "sides" of the chromomere from which the loop ends emerge, hence the existence of double bridges, and diffentiate between the chromatin of identical chromatids, hence the existence of double-axis regions. Hence, my schematic representation of the four-partite chromomere in Fig. 4.

All rather vaguely hypothetical, you will say! Well now it is time to enter the second great era of lampbrushology and look at the molecular and biophysical properties of the loop/chromomere complex.

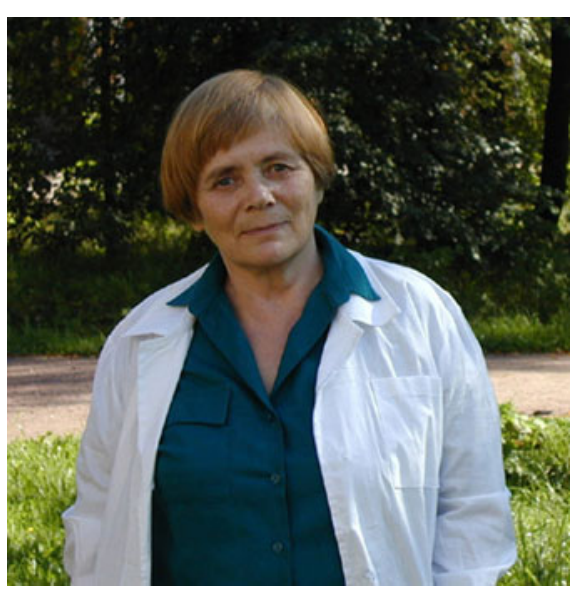

Fig. 10 Elena Gaginskaya, photographed in 2000

\section{The avian $\mathrm{W}$ chromosome}

It was during the 1980s in Soviet Russia that Elena Gaginskaya (Fig. 10) started to look at the lampbrush chromosomes of birds and her extraordinary skill and initiative in working with this technically challenging system generated a whole new perspective for lampbrushology (Gaginskaya et al. 2009). To begin with, she looked at the sex chromosomes because only in birds is it possible to see both members of a heteromorphic sex chromosome pair-the $\mathrm{Z}$ and $\mathrm{W}$ - in the lampbrush form.

The chicken Z/W lampbrush chromosome pair looks like a univalent (Solovei et al. 1993, 1998; Mizuno and Macgregor 1998). Most of it has a typical lampbrush organisation but the terminal one fifth consists of a relatively thick axis carrying only a very few small lateral loops (Fig. 11). The region with normal lampbrush appearance is the $\mathrm{Z}$ chromosome; the short thick loopless region is the $\mathrm{W}$ (indicated by arrow $\mathrm{W}$ in Fig. 11). The point at which these two chromosomes are joined by a single chiasma (C in Fig. 11) is usually marked by two adjacent pairs of large conspicuous loops, one belonging to the end of the $\mathrm{W}$ and the other to the end of the $\mathrm{Z}$ (Fig. 11).

The chicken W-LBC is $10-15 \mu \mathrm{m}$ long. Its axis consists of six or seven densely packed chromomeres that are larger and brighter (after staining with DAPI) than any of the chromomeres on the $\mathrm{Z}$ chromosome. The arrangement of chromomeres on the $\mathrm{W}$ is absolutely consistent (Fig. 12) and each chromomere is individually recognisable. W chromosomes with seven chromomeres have a very bright chromomere at the extreme free end of the chromosome; this is missing in 
Fig. 11 The ZW lampbrush bivalent of the chicken. The short condensed W chromosome is marked with an arrow $(W)$. The two pairs of large conspicuous loops, one of them open-ended, mark the site of the terminal chiasma $(C)$ that joins the $Z$ and $W$ together to form a bivalent

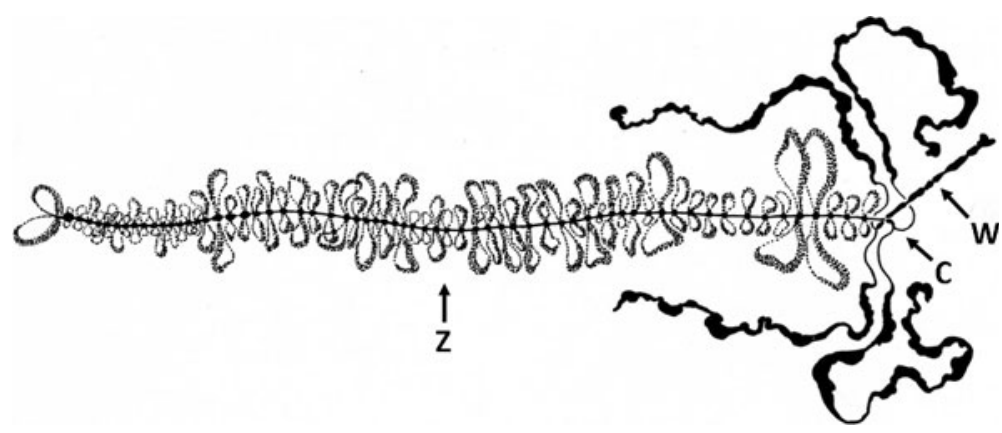

Ws with six chromomeres. It is impossible to say that the $\mathrm{W}$ chromosome is loopless. It has a very few conspicuous loops and varies in this respect from one species of bird to another. What we do not know is if it has any little loops that cannot be seen in the light microscope - but I would like to think that it does not.

The W sex chromosome heterochromatin is partly made up of two repetitive DNA sequence familiesthe EcoRI and XhoI. Both families consist of tandem repeats of a basic unit of about $21 \mathrm{bp}$. These two

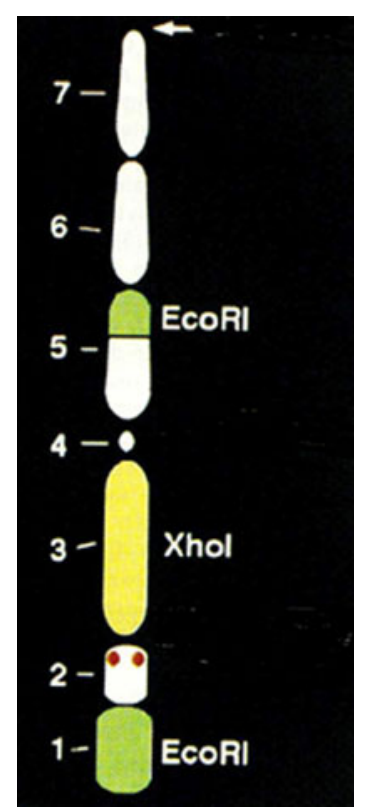

Fig. 12 Arrangement of the seven chromomeres of the chicken $\mathrm{W}$ chromosome. The arrow at the top indicates the chiasmate end of the chromosome that is attached to the Z. Chromomere 1 is especially bright when stained with DAPI and is absent in some individuals. EcoR 1 and Xho 1 indicate the chromomeres that are made up entirely, if not exclusively of the corresponding repeated sequences. Diagram extracted from Ogawa et al. (1997) families are not intermingled with one another. The XhoI family consists of about 30,000 repeats; the EcoRI family varies between 700 and 4,000 repeats (Solovei et al. 1998; Mizuno and Macgregor 1998).

In situ hybridisation experiments have shown with astonishing clarity that the XhoI repeat family hybridises exclusively to small chromomere number 3 on the W chromosome and the EcoRI family hybridises exclusively to the proximal part of chromomere 5 and the distal part of chromomere 1 (Solovei et al. 1998). These are the only examples to date of single families of repeated DNA sequences being localised in specific chromomeres.

\section{Centromeres and chromomeres}

In places where we would expect to find lampbrush centromeres, there is usually a small loopless granule on or slightly to the side of the chromosome axis. I am deliberately avoiding the word chromomere here because the nature of this centromere granule remains something of an enigma. It looks featureless in scanning electron micrographs of fixed and critical pointdried LBCs. In transmission electron micrographs of thin sections of plastic-embedded LBCs, it again appears as a featureless homogeneous mass of what seems like compact chromatin. As expected, there are no kinetochores associated with these "centro-chromomeres". No surprises - and no thrills- here.

A group of Japanese scientists recently identified centromeric protein A (CENP-A) interacting DNA sequences in chicken genome (Shang et al. 2010), amongst which are several chromosome-specific centromere repeats (Cen1, Cen2, Cen3, Cen4, Cen7, Cen8 and Cen11). These repeats have a clear function in kinetochore formation. Krasikova and her coworkers (see Krasikova and Gaginskaya 2010 and 
Fig. 13 The middle section of one of the lampbrush bivalents from a crested newt, Triturus cristatus karelinii showing the centromere granule (arrows) flanked by two large bars of dense loopless chromatin. Drawing by Lydia Lloyd

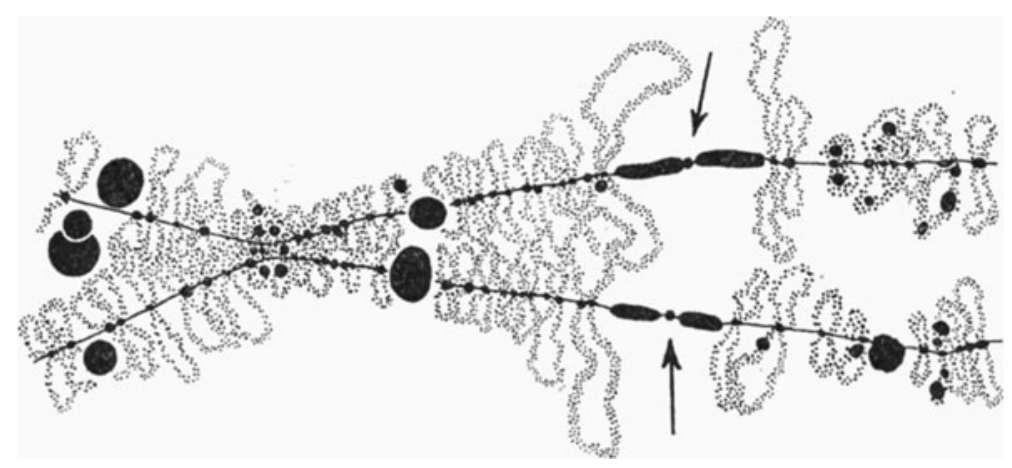

Krasikova et al. ibid.) have also identified tandem DNA repeats that interact with CENP-A in the vicinity of the centromere of bird chromosomes in somatic cells. Neocentromeres and immature centromeres do not contain these satellite repeats, but do have some non-tandemly repetitive DNA that interacts with CENP-A. During the lampbrush stage, CENP-A protein is not detectable at centromeres of lampbrush chromosomes but it may be reasonable to define the position of a lampbrush centromere by the presence of centromere repeats that are known to interact with CENP-A at other stages of cell cycle and in other tissues (see Krasikova et al. ibid.).

The situation becomes more complex and intriguing when protein bodies enter the discussion. The protein body (PB), first described by Elena Gaginskaya and her colleagues in St. Petersburg (Gaginskaya 1972; Saifitdinova et al. 2003; Krasikova et al. 2005), is a locus-specific marker that shows highly conserved synteny at centromere regions of lampbrush chromosomes in all birds studied so far and several amphibian species. It can be unequivocally identified on the basis of its distinctive appearance and by marker components, including sub-units of the cohesin complex. The exact role of the PB remains unknown although several reasonable suggestions have been offered.

As with the centromere PBs of birds, the centromere granule of amphibian LBCs often sits between two large chromomeres in centromere regions (Fig. 13). In some species of urodele, the lampbrush centromeres are flanked by dense bars of loopless chromatin. These were first described by Callan and Lloyd (1960) and an example of one of Lloyd's beautiful and highly accurate camera-lucida drawings is shown in Fig. 13 along with a typical example of this kind of situation in phase contrast (Fig. 14).
The DNA in these "centromere bars" consists almost entirely of two highly repeated short DNA sequences. One of them is $33 \mathrm{bp}$ long, makes up nearly $10 \%$ of the entire genome and is repeated about eight million times. The other is $68 \mathrm{bp}$ long, is not a simple dimer of the first and is repeated about four million times. Both are detectable exclusively in the pericentromeric heterochromatin of all chromosomes and in the centromere bars of all lampbrushes (Baldwin and Macgregor 1985). So here again, we have a compact "mega-chromomere" that consists largely, if not entirely, of one or a very few DNA sequence families. We do not know if these DNA sequences have any affinity for CENPs but it is undoubtedly something that should be investigated.

According to Gaginskaya and Krasikova and their colleagues, the thing that we call the "centromere granule" in amphibians should be a PB (Gaginskaya et al. 2009; Krasikova and Gaginskaya 2010). Fluorescence in situ hybridization mapping of an RrS1 repeat on LBCs of green frogs ( $\mathrm{Rr}=$ Rana (now Pelophylax) ridibundus) shows that this repeat localizes in two objects that surround the "centromere granule". Moreover, after immufluorescent staining with antibodies against histone modifications typical for heterochromatin (histone H3 K27diMe), no signal can be detected in the "centromere granules" (see Krasikova et al. ibid.).

I suggest that we can conclude from all these observations that somewhere in the centromere regions of LBCs, there are CENP-interacting DNA sequences that are not condensed into the form of a visible chromomere, that there are nearby some DNA sequences that play a role in the formation of the PB and that certain components of the PB have an important role in the behaviour of the centromeric region in relation to kinetochore formation and sister and/or homologous chromatid cohesion. That is probably about as far as we can go at the moment but at least in the 


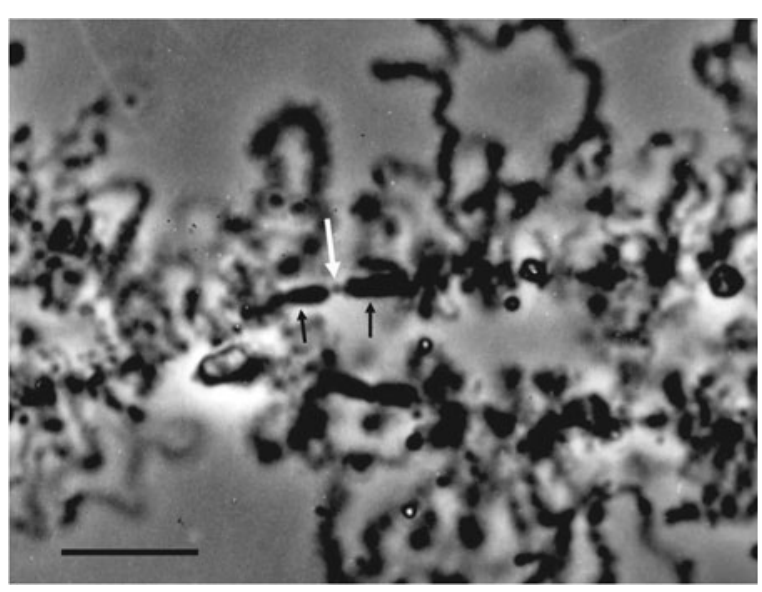

Fig. 14 Phase contrast micrographs of the centromere region of a lampbrush bivalent from a newt showing the "centromere granule" (large white arrow) flanked by two dense bars of pericentromeric heretochromatin (smaller black arrows). The scale bar represents $20 \mu \mathrm{m}$

context of this essay, we can say that there seems to be no evidence for a lampbrush, centromeric DNAcontaining chromomere. Tantalisingly curious because, although centromeres are an irrelevance during the lampbrush stage, they and all their complex machinery will suddenly become very important indeed as first meiotic metaphase approaches.

\section{Telomeres}

The ends of lampbrush chromosomes were even less interesting than their centromeres until the Russian lampbrushologists got to work on birds. Callan and Lloyd refrain from mentioning telomeres in their 1960 paper and cautiously represent them in some of their otherwise meticulously detailed drawings as tiny loopless terminal dots.

So once again, the most telling observations came from birds. In most of these, LBCs end in a terminal chromomere with a pair of loops (Solovei et al. 1994, 1995) and quite often the telomere loops are open ended, the loop having one insertion into the chromomere whilst the other end hangs free (Figs. 15 and 16). Telomeric loops in chicken usually consist of two transcription units the most terminal of which is $0.5-$ $15 \mu \mathrm{m}$ long and consists entirely of the telomeric sequence TTAGGG. Transcription is from the C-rich strand and proceeds outwards towards the open end of the loop. So here, the chromosome ends at the end of a transcription unit, not at a chromomere. But does the fact that occasionally the telomere is stitched back onto main body of the chromosome, as in the upper half bivalent on Fig. 14, suggest that that the chromosome may sometimes end in some non-transcribed, non-telomeric DNA sequences that are disposed to remain loosely bound to a small mass of compacted sub-terminal chromatin-a chromomere?

\section{Molecules and all that}

To proceed any further, we have ask questions that can only be answered through the use of modern molecular probes and here are some answers.

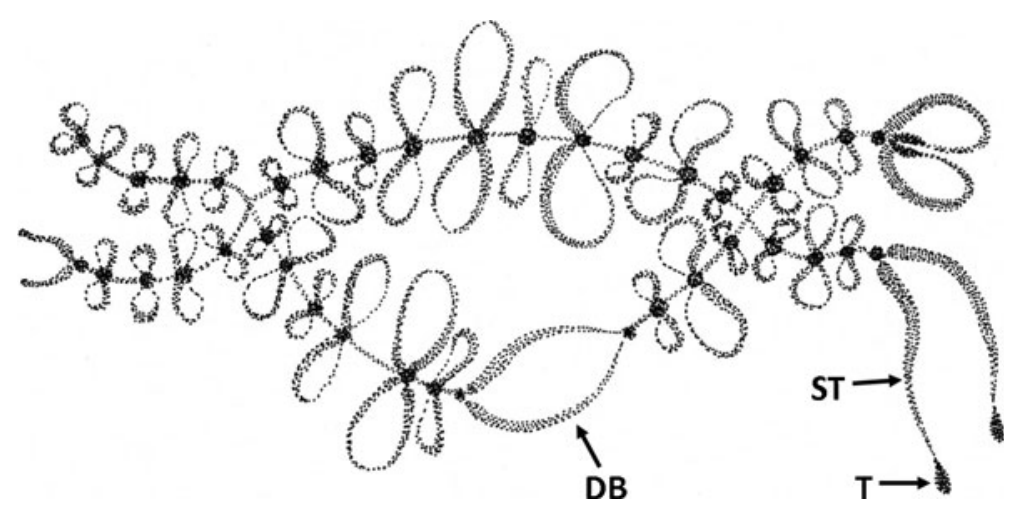

Fig. 15 Drawing of part of a lampbrush bivalent from a chicken showing the large terminal loops at the right hand end of each of the two half-bivalents. In the upper one, the loops are attached to the chromosome at both ends. In the lower one, the terminal loops hang free and show two transcription units, the inner of which $(S T)$ has a distal-proximal polarity and short outer or terminal transcription unit $(T)$ has a proximal-distal polarity. The lower of the two half bivalents shows a double bridge $(D B)$ 


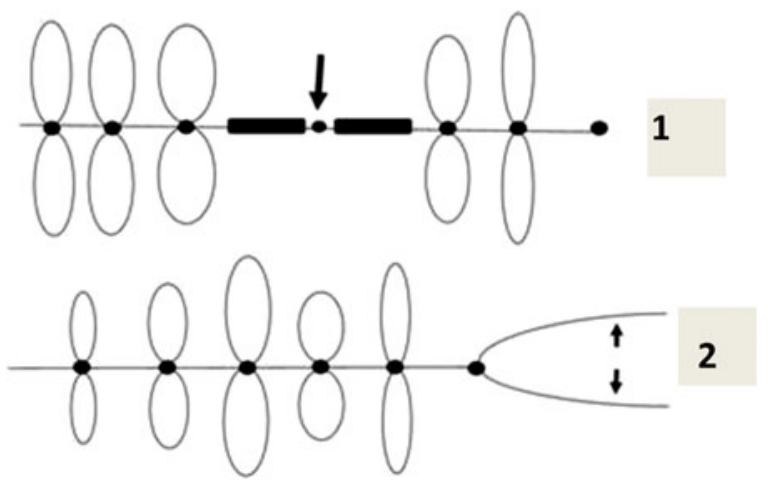

Fig. 161 Loopless "chromomere" marking the position of the centromere (arrow), with chromatin bars. 2 Avian LBC with typical open-ended telomeric loop

Somatic Histone H1, which is implicated in the formation of higher order chromatin structures and associated with transcription repression, is absent from LBCs in yolky oocytes where transcription is at its maximum level but only just detectable in the small, transparent pre-vitellogenic oocytes where transcription is not yet quite up to speed (Hock et al. 1993). Not entirely unexpected!

Cohesin is associated with the axis but not the loops and shows a high mobility between nucleoplasm and the chromosome (Austin et al. 2009; Krasikova et al. 2005). A most revealing observation!

SMC3 (a component of the multimeric cohesin complex that holds sister chromatids together) and hRAD21 (a nuclear phospho-protein with a role in sister chromatid cohesion) are present at high levels in the chromomeres (Austin et al. 2009). Good, since if they were not there, we would have to wonder what held chromomeres together at all!

Condensin component XCAP-D2 is present at high levels in the chromomeres (Beenders et al. 2003). Heterochromatin protein HP1 $\beta$ accumulates in chromomeres with a high concentration of tandem repeats (Krasikova et al. 2009).

5-Methylcytosine is located in transcriptionally inactive chromatin in the lampbrush chromosome axis and in the untranscribed DNP-spacers of some loops (Angelier et al. 1986).

Trimethylation of histone $\mathrm{H} 3$ at lysine 27 (H3K27me3) is typical for all chromomeres, being abundant in the regions of "constitutive" heterochromatin (Krasikova et al. 2009).

Non-repressive modification is associated with the transcription units on laterally projecting loops
(Krasikova et al. 2009). Histone H4 is hyperacetylated in the regions of attachment of loops to chromomeres (Sommerville et al. 1993) which may signify a loosening of the chromatin in those places, making it more accessible to transcriptional proteins, and there are acetylated histones at particular points ("nodes") in lateral loops, which have been interpreted as likely regions of incompletely decondensed chromatin (Gaginskaya et al. 2009).

The embryonic histone H1M variant (also known as B4), another chromatin modifying protein is absent from LBCs (Korner et al. 2003) - satisfyingly so, since its presence would be inconsistent with other molecular information and would complicate matters considerably; but unlike somatic $\mathrm{H} 1$, it is a prominent component of the non-chromatinised, maternal store of histones in the oocyte.

Other interesting LBC absentees are the architectural chromatin proteins, HMGN (Korner et al. 2003). Injection of $\mathrm{H} 1$ into oocytes shuts down transcription and causes loop retraction and chromosome shortening (Hock et al. 1993). A helpful observation!

DNA topoisomerase II, a major component of the scaffold of metaphase chromosomes, is absent from lampbrush chromatin but present in axial granules (categorised as chromomere-associated structures; Hock et al. 1996; Krasikova et al. 2004). Again, hardly surprising, although its presence in axial granules remains unexplained.

A final point about lampbrush chromatin that I think will become increasingly important is the suggestion from some recent work of John Sommerville's laboratory that LBC-associated histones are continually being exchanged with free histones, further indication of the highly dynamic chromatin environment in lampbrush stage oocytes (Stewart et al. 2006).

\section{Conclusions}

For a double bridge to form, the two chromatids must have remained in register with one another since the pre-meiotic replication-not surprising - and the chromatin must be sufficiently loose as to allow it to part into two masses to which the ends of the loop are attached. If it were compacted, the tension that would normally produce a DB break would probably break the interchromomeric fibril. Herein may lie a selective advantage of absence of $\mathrm{H} 1$ and SMC2. 
DB breaks probably only happen where there is a very long loop that may represent more than the average proportion of loop/chromomere DNA. So the question of how the four segments of the chromomere are kept so effectively apart actually only applies to situations where a large transcription unit (or contiguous series of units) comes from a small chromomere. They are not kept apart; they just never get mixed up — rather like the hypothetical arrangement depicted in Fig. 4.

Some chromomeres have lots of loops, including micro-loops that are not discernible with the light microscope (Gaginskaya and Tsvetkov 1988; Krasikova, unpublished observations). So the chromomere/loop pair principle that dates back more than 60 years is no longer so important. Chromomeric chromatin is where there is no transcription; if it is not transcribing, it condenses loosely to form what we see and call a chromomere.

Despite the reasonable assumption that chromomere pattern might be inherently regular, as in polytene chromosomes, in LBCs it is so dynamic that the pattern is rarely evident.

To his great credit, Callan's 30-year-old (Callan 1982) explanation is consistent with all the observed facts and modern data.

When neighbouring transcription units of $L B C S$ lie far enough apart, the untranscribed intervening regions condense into chromomeric DNP, and multiple pairs of loops will extend from aggregate chromomeres provided that their RNP transcripts are not particularly bulky. However, when the transcripts at the termini of transcription reach a critical bulk, they disrupt such aggregates of DNP into smaller chromomeres with which fewer or single loop pairs are associated, and such chromomeres are forced to maintain their continuity with one another by extending lengths of untranscribed DNP, the interchromomeric strands, until transcription shuts off, when recompaction occurs leading up to meiotic metaphase.

In other words, LBCs are a physically induced phenomenon, facilitated by the selective absence of molecular factors that would interfere with their main transcriptional role. LBC morphology is highly dynamic and driven by transcriptive activity.

The key to the whole situation is the incomplete compaction and dynamic nature of lampbrush chromatin. This not only faciltiates transcription but keeps the chromosome safe from changes that might impede its orderly progression through meiotic metaphase 1arguably, the most important event in the entire life of an organism. The physical problems that would ensue in the closing stages of meiotic prophase if the chromosomes had been allowed to decondense completely after synapsis and crossing over are unimaginable! For reasons that, amazingly, we do not yet fully understand, transcription has to take place at thousands of loci during an extended diplotene in the female gametes of most animals except mammals and lampbrush chromosomes represent a mechanism that has evolved to ensure that this transcription can happen without compromising the success of meiosis.

It is all really quite simple and the evolutionary sequence is plain to see. Half a century later, my mind is at rest!

\section{References}

Angelier N, Bonnanfant-Jais ML, Moreau N, Gounon P, Lavaud A (1986) DNA methylation and RNA transcriptional activity in amphibian lampbrush chromosomes. Chromosoma 94:169-182

Austin C, Novikova N et al (2009) Lampbrush chromosomes enable study of cohesin dynamics. Chromosom Res 17:165-184

Baldwin L, Macgregor HC (1985) Centromeric satellite DNA in the newt Triturus cristatus karelinii and related species: its distribution and transcription on lampbrush chromosomes. Chromosoma 92:100-107

Beenders B, Watrin E, Legagneux V et al (2003) Distribution of XCAP-E and XCAP-D2 in the Xenopus oocyte nucleus. Chromosom Res 11:549-564

Callan HG (1957) The lampbrush chromosomes of Sepia officinalis L., Anilocra physodes L. and Scyllium catulus Cuv. and their structural relationship to the lampbrush chromosomes of Amphibia. Pubbl Stn zool Napoli 29:329-346

Callan HG (1982) Lampbrush chromosomes. Proc R Soc London, Ser B 214:417-448

Callan HG (1986) Lampbrush chromosomes. Springer, Berlin

Callan HG, Lloyd L (1956) Visual demonstration of allelic differences within cell nuclei. Nature 178:355-357

Callan HG, Lloyd L (1960) Lampbrush chromosomes of crested newts Triturus cristatus (Laurenti). Philos Trans R Soc London Ser B 243:135-219

Gaginskaya ER (1972) The nuclear structures in oocytes of adult birds. II. Protein bodies and the karyosphere. Tsitologiia 14:568-577

Gaginskaya ER, Tsvetkov AG (1988) Electron microscopy research on the chromatin structure of dispersed lampbrush chromosomes in the hen. Tsitologia 30:142-150

Gaginskaya E, Kulikova T, Krasikova A (2009) Avian lampbrush chromosomes - a powerful tool for the exploration of gene expression. Cytogenetic and Genome Research 124:251-267 
Gall JG (1963) Kinetics of deoxyribonuclease action on chromosomes. Nature 198:36-38

Gall JG, Murphy C (1998) Assembly of lampbrush chromosomes from sperm chromatin. Mol Biol Cell 9:733-747

Hill RS, Macgregor HC (1980) The development of lampbrush chromosome-type transcription in early diplotene oocytes of Xenopus laevis: an electron microscope analysis. J Cell Sci 44:87-101

Hock R, Moorman A, Fischer D, Scheer U (1993) Absence of somatic histone $\mathrm{H} 1$ in oocytes and preblastula embryos of Xenopus laevis. Dev Biol 158:510-522

Hock R, Carl M et al (1996) A monoclonal antibody against DNA topoisomerase II labels the axial granules of Pleurodeles lampbrush chromosomes. Chromosoma 104:358-366

Korner U, Bustin M, Scheer U, Hock R (2003) Developmental role of HMGN proteins in Xenopus laevis. Mech Dev 120:1177-1192

Krasikova A, Gaginskaya E (2010) Organization of centromere regions of chromosomes in the lampbrush phase. Tsitologia 52(7):515-533

Krasikova A, Kulikova T, Saifitdinova A, Derjusheva S, Gaginskaya E (2004) Centromeric protein bodies on avian lampbrush chromosomes contain a protein detectable with an antibody against DNA topoisomerase II. Chromosoma 113:316-323

Krasikova A, Kulikova T, Saifitdinova A (2005) Cohesion proteins are present in centromere protein bodies associated with avian lampbrush chromosomes. Chromosom Res 1:675-685

Krasikova A, Daks A, Zlotina A, Gaginskaya E (2009) Polymorphic heterochromatic segments in Japanese quail microchromosomes. Cytogenetic and Genome Research 126:148-155

Macgregor HC (1972) The nucleolus and its genes in amphibian oogenesis. Biol Rev 47:177-210

Macgregor HC (1978) Trends in the evolution of very large chromosomes. Proc Roy Soc London B283:309-318

Mizuno S, Macgregor HC (1998) The ZW lampbrush chromosomes of birds: a unique opportunity to look at the molecular cytogenetics of sex chromosomes. Cytogenet Cell Genet 80:149-157

Mott MR, Callan HG (1975) An electron-microscope study of the lampbrush chromosomes of the newt Triturus cristatus. J Cell Sci 17:241-261

Ogawa A, Solovei I, Hutchison N, Saitoh Y, Ikeda J-E, Macgregor H, Mizuno S (1997) Molecular characterization and cytological mapping of a non-repetitive DNA sequence region from the $\mathrm{W}$ chromosome of chicken and its use as a universal probe for sexing Carinatae birds. Chromosom Res 5:93-101
Saifitdinova A, Derjusheva S, Krasikova A, Gaginskaya E (2003) Lampbrush chromosomes of the chaffinch (Fringilla coelebs L.). Chromosom Res 11:99-113

Scheer U, Franke W, Trendelenburg MF, Spring H (1976) Classification of loops of lampbrush chromosomes according to the arrangement of transcriptional complexes. J Cell Sci 22:503-519

Sessions SK (2008) Evolutionary cytogenetics in salamanders. Chromosom Res 16:183-201

Snow MHL, Callan HG (1969) Evidence for a polarized movement of the lateral loops of newt lampbrush chromosomes during oogenesis. J Cell Sci 5:1-25

Solovei I, Gaginskaya E, Hutchison N, Macgregor HC (1993) Avian sex chromosomes in the lampbrush form: the ZW lampbrush bivalents from six species of bird. Chromosom Res 1:153-166

Solovei I, Gaginskaya ER, Macgregor HC (1994) The arrangement and transcription of telomere DNA sequences at the ends of lampbrush chromosomes of birds. Chromosom Res 2:460-470

Solovei I, Macgregor H C and Gaginskaya E (1995) Specifically terminal clusters of telomere DNA sequences are transcribed from the C-rich strand on chicken lampbrush chromosomes. PF Brandham and MD Bennett (eds). Proc. Kew Chromosome Conference IV. Royal Botanic Gardens: Kew, UK. pp 323-330

Solovei I, Ogawa A, Naito M, Mizuno S, Macgregor HC (1998) Specific chromomeres on the chicken W lampbrush chromosome contain specific repetitive DNA sequence families. Chromosom Res 6:323-327

Sommerville J, Baird J et al (1993) Histone H4 acetylation and transcription in amphibian chromatin. J Cell Biol 120:277290

Stewart MD, Sommerville J et al (2006) Dynamic regulation of histone modifications in Xenopus oocytes through histone exchange. Mol Cell Biol 26:6890-6901

Vlad M, Macgregor HC (1975) Chromomere number and its genetic significance in lampbrush chromosomes. Chromosoma 50:327-347

Wei-Hao S, Tetsuya H, Atsushi T et al (2010) Chickens possess centromeres with both extended tandem repeats and short non-tandem-repetitive sequences. Genome Res 9:12191228

White MJD (1954) Animal cytology and evolution. The University Press, Cambridge UK

Wilson EB (1925) The cell in development and heredity. Macmillan, New York 